ISSN 0103-5150

Fisioter. Mov., Curitiba, v. 25, n. 2, p. 435-443, abr./jun. 2012 Licenciado sob uma Licença Creative Commons

\title{
Exercício resistido em idosos frágeis: uma revisão da literatura
}

\author{
Resistance exercise in frail elderly: a literature review
}

\author{
Lucas Caseri Câmara $^{[a]}$, Carina Corrêa Bastos ${ }^{[b]}$, Esther Fernandes Tinoco Volpe ${ }^{[c]}$ \\ [a] Mestrando em Ciências (Fisiopatologia Experimental), Universidade de São Paulo (USP), São Paulo, SP - Brasil, e-mail: \\ lucasccmed@hotmail.com \\ [b] Mestranda em Gerontologia Biomédica, Pontifícia Universidade Católica do Rio Grande do Sul (PUCRS), Porto Alegre, RS - \\ Brasil, e-mail: cacabastos@hotmail.com \\ [c] Mestranda em Cardiogeriatria pela Universidade Federal de São Paulo (Unifesp), São Paulo, SP - Brasil, e-mail: \\ esthertinoco@yahoo.com.br
}

\section{Resumo}

Introdução: A síndrome da fragilidade, bastante comum em pessoas de idade avançada, consiste em um conjunto de sinais e sintomas no qual estão presentes critérios como perda de peso corporal não intencional em um ano (aproximadamente 5\%), diminuição na velocidade da marcha, níveis baixos de atividade física, exaustão subjetiva e diminuição de força muscular. Os consequentes efeitos dessas mudanças relacionadas à idade, que incluem sarcopenia, disfunção imunológica e desregulação neuroendócrina, aumentam a vulnerabilidade do organismo ao estresse, reduzindo a habilidade de adaptar, compensar ou modular esses estímulos. Diferentes intervenções têm sido propostas para atenuar esse processo, sendo o exercício resistido (ER) uma das opções estudadas. Objetivo: Realizar uma revisão bibliográfica averiguando os efeitos dos ER na fisiopatologia da síndrome da fragilidade. Materiais e métodos: Foi realizada uma revisão bibliográfica do período de 2004 a 2010, por meio das bases de dados LILACS, MEDLINE e PubMed. Resultados: Por meio das análises dos estudos, foram observadas alterações nos sistemas hormonal e imune, atuando de forma sistêmica na reversão ou minimização dos efeitos da sarcopenia exercendo influência positiva na síndrome da fragilidade. Conclusão: 0 ER deve ser indicado como opção terapêutica para idosos frágeis ou pré-frágeis que não apresentem contraindicações para realização desta modalidade de exercício.

Palavras-chave: Idoso débil. Levantamento de peso. Terapia por exercício. 


\section{Abstract}

Introduction: Fragility syndrome, very common in elderly people, consists of a set of signals and symptoms in which is present criteria such as not intentional weight loss (approximately 5\%) in a year, reduction in the walking speed, low physical activity levels, subjective exhaustion and muscular strength reduction. The increasing effect of these changes related to age, which include sarcopenia, immunity functional disorder and neuroendocrinous misconduct, increase the vulnerability of the organism to stress, reducing the ability to adapt, compensate or modulate these stimuli. Several intervention proposals have been made to attenuate this process, and resistance exercises (RE) was one of the options studied. Objectives: To evaluate the effects of RE on the physiopathology of fragility syndrome. Materials and methods: A bibliographic review of the period 2004-2010 was made based on the data of LILACS, MEDLINE and PubMed. Results: Changes in the hormonal and immune systems were observed acting in a systemic way by reverting or minimizing the effects of sarcopenia. Conclusion: Resistance exercises should serve as therapy to those elderly who are fragile and do not present any health problems.

Keywords: Frail elderly. Weight lifting. Exercise therapy.

\section{Introdução}

A síndrome da fragilidade, já consagrada em diversos países, caracteriza-se por apresentar pelo menos três de cinco critérios: diminuição da força muscular, baixo gasto energético, diminuição da velocidade de marcha, perda de aproximadamente $5 \%$ do peso corporal de forma involuntária em um ano e exaustão subjetiva (1). A base biológica dessa síndrome consiste na diminuição da reserva e da resistência a estresses físicos, caracterizadas por um alto grau de vulnerabilidade para incapacidade, comorbidades, quedas, hospitalização, institucionalização e morte (1-4). Os aspectos da fragilidade incluem, ainda, redução na mobilidade, anormalidade na marcha, fraqueza muscular, tolerância reduzida ao exercício, equilíbrio instável, má nutrição e sarcopenia $(1,5)$. Sua prevalência nos Estados Unidos é de quase 7\% na comunidade (1). No Brasil, ainda não temos dados epidemiológicos baseados em instrumentos com propriedades psicométricas validadas.

A complexidade da fragilidade dificulta a identificação de fatores biológicos que a justifique. Essa situação é agravada pelas múltiplas comorbidades normalmente observada em idosos. Os investigadores supõem que as várias alterações relacionadas ao envelhecimento, genética e doenças contribuem para a fisiologia alterada encontrada na fragilidade. Os sistemas mais proeminentes em estudos da fisiopatologia da fragilidade incluem o músculo esquelético, endócrino e sistema imunológico (6).

De acordo com Morley et al. (7), assim que diagnosticada a síndrome da fragilidade, deve-se iniciar a intervenção com exercícios resistidos (ER) e de equilíbrio. Segundo recente revisão sistemática a respeito das técnicas convencionais utilizadas na prática fisioterapêutica (exercícios de equilíbrio, flexibilidade, coordenação, força e tempo de reação), os autores concluíram que apesar de terem sido encontrados ganhos significativos em relação à força, equilíbrio e capacidade funcional, não foi possível, com as intervenções utilizadas, reverter ou impedir a progressão da fragilidade (8).

O ER (caracterizado pela realização de contrações musculares contra alguma forma de resistência, em geral pesos) vem ganhando destaque na comunidade científica, por sua segurança e eficácia, mesmo para indivíduos doentes ou debilitados $(9,10)$. Essa modalidade de exercícios contribui para o aumento da massa e melhora da força muscular, e é atualmente recomendado por renomadas organizações como atividade de promoção de saúde (11-13), melhorando a força muscular, a capacidade aeróbica e o equilíbrio, reduzindo e retardando, assim, a fragilidade e a dependência física $(4,14)$.

Nos últimos anos, a consagração da SF vem sendo crescentemente descrita por diversos autores. A identificação dessa síndrome nos idosos, por meio de instrumentos de aplicabilidade mais eficiente, também tem sido discutida. Porém, as modalidades de tratamento para reverter ou minimizar seus efeitos ainda não têm sido suficientemente publicadas. Os efeitos terapêuticos do ER caminham em sentido contrário ao das alterações degenerativas observadas na fragilidade (15). No entanto, a prática dessa modalidade de exercícios ainda se apresenta pouco estudada com 
relação a essa síndrome especificamente. Alguns estudos associam efeitos dos exercícios nos resultados adversos de idosos com síndrome da fragilidade, mas especificamente a sua influência na fisiopatologia não tem sido discutida com maior profundidade, fato que fomentou a realização deste estudo. Assim, o objetivo desta revisão foi reunir os estudos que apontaram os efeitos da prática regular dos ER nas características fisiopatológicas da síndrome da fragilidade: sarcopenia, disfunção neuroendócrina e imunológica, bem como buscar um modelo possivelmente adequado para prescrição nesta população.

\section{Métodos}

Foi realizado um levantamento literário de estudos nacionais e internacionais que se referem ao tema, encontrados nas bases de dados LILACS, MEDLINE e PubMed com publicação entre os anos de 2004 e 2010. Esta seleção foi feita em virtude do fato de a síndrome da fragilidade ter sido descrita de forma mais consistente (critérios de definição mais compatíveis com os da atualidade) nos estudos a partir de 2004. Foram considerados, pelos autores, aqueles estudos que relacionavam a influência do exercício resistido nas características fisiopatológicas encontradas em idosos com SF. Para tanto, foram utilizadas para a pesquisa as seguintes palavras-chave (na língua portuguesa e inglesa): idosos, fragilidade, exercício resistido, sarcopenia, disfunção neuroendócrina e imunológica.

Dentre as referências encontradas, foram selecionados 40 artigos julgados relevantes, incluindo estudos experimentais e revisões. A busca dos periódicos completos foi realizada nos portais científicos de revistas eletrônicas "Portal da pesquisa", "SIBI", e "OVID". Já a pesquisa por trabalhos completos foi feita nas Bibliotecas da Faculdade de Medicina da Universidade de São Paulo e na Pontifícia Universidade Católica do Rio Grande do Sul (PUCRS).

\section{Resultados}

\section{Exercícios resistidos e sarcopenia}

Sarcopenia, termo proposto por Rosenberg em 1989, refere-se especificamente à perda de massa muscular associada ao envelhecimento. No entanto, esse termo tem sido estendido à perda de força muscular relacionada com o avançar da idade $(16,17)$.

A prevalência de sarcopenia é de aproximadamente $12 \%$ para adultos de 60 a 70 anos de idade, aumentando para $30 \%$ por volta dos 80 anos de idade. Na maioria dos estudos, o seu desenvolvimento está fortemente associado à elevada incapacidade, desordens na marcha e no equilíbrio e mortalidade (7).

A causa da sarcopenia é multifatorial, resultante de alterações no sistema nervoso (perda de unidades motoras alfa), musculares (perda na qualidade e massa muscular), hormonais (diminuição de hormônios anabolizantes, como testosterona, estrógeno e GH) e estilo de vida (diminuição da atividade física) (18).

Por meio da prática regular de exercícios físicos, dentre os quais os ER se destacam, pode ser viável prevenir a sarcopenia (19) e melhorar consistentemente a força em idosos (20).

Estudos demonstraram aumento da síntese de proteínas miofibrilares musculares em jovens e idosos por meio dos ER (19). De acordo com uma revisão sistemática sobre intervenções para sarcopenia (15), o ER foi considerado o estímulo mais poderoso para a hipertrofia muscular, quando comparado aos exercícios contínuos. Os autores citam, ainda, que em comparação a sujeitos jovens, o ER em pessoas idosas produz aumento de força menor em termos absolutos, mas similares em termos relativos. Ganhos de 5-10\% na área de seção transversal muscular acompanhada por aumento de $20 \%$ a $100 \%$ na força muscular, dependendo do grupo de músculos, devem ser expectativas razoáveis de um regime apropriado de exercícios (21).

Um estudo utilizando 25 idosos saudáveis e 26 adultos jovens verificou uma diminuição da força muscular e da expressão genética mitocondrial (maior fator contribuinte da sarcopenia) dos idosos em relação aos jovens. Os indivíduos jovens apresentaram, inicialmente, um pico de torque $59 \%$ maior que os idosos saudáveis e, após seis meses de treinamento resistido, essa diferença diminuiu para 38\%. Por meio de biópsia no vasto lateral dos indivíduos, observou-se também uma modificação no perfil de expressão genética mitocondrial (jovialização desse perfil) nos músculos dos idosos saudáveis submetidos a treinamento resistido (22). Embora a resposta à hipertrofia seja reduzida em idosos, ocorre um aumento na qualidade muscular (performance muscular) de maneira similar entre homens idosos e jovens; porém, pode ser maior em mulheres jovens do que em idosas (23). 
Os ganhos na força muscular podem ser observados nas primeiras semanas, sendo atingido um platô por volta de 5-6 meses. Esses ganhos na força refletem adaptações neurais e musculares, com hipertrofia de fibra muscular, tornando-se dominante com duração de treino prolongado (24).

Vários estudos demonstram ganho de percentagem similar de força entre participantes idosos e jovens, enquanto outros têm mostrado que o aumento na percentagem de força é menor para idosos quando comparados a adultos jovens. Estudos adicionais sugerem que o efeito da idade na adaptação da força pode ser influenciado pelo sexo, duração do treinamento e/ou por grupos musculares específicos examinados (23).

Em um estudo com homens e mulheres frágeis da comunidade que realizaram um programa de exercícios resistidos de baixa a moderada intensidade, observou-se aumento na força muscular isocinética e aumento na massa magra local e total (25).

\section{Exercícios e disfunção neuroendócrina e imunológica}

Estudos epidemiológicos demonstram que a função imune, a qual se encontra prejudicada no envelhecimento, contribui para o aumento da susceptibilidade a infecções e, também, está associada a várias causas de mortalidade $(26,27)$. 0 exercício físico (especialmente o moderado) (28), considerado como um protótipo de estresse que pode ser controlado experimentalmente, induz melhora significativa no sistema imune e pode ser particularmente benéfico para aquelas pessoas com a resposta imunológica deficiente, como os idosos frágeis (27).

As células do sistema imune que mostram mais resposta aos efeitos do exercício intenso e prolongado, tanto em termo de número como de função, são as células NK, neutrófilos e macrófagos $(29,30)$. Um estudo comparou idosos que se exercitam e idosos sedentários, com o objetivo de observar se o treinamento cardiovascular poderia melhorar a resposta imune na vacinação do vírus influenza. 0 grupo exercitado apresentou resposta aumentada de anticorpos para vacinação em relação ao outro grupo, porém não foram observadas diferenças entre os grupos quanto às células mediadoras para resposta imune (31).

0 exercício físico moderado e regular tende a reduzir sintomas de infecções do trato respiratório, em contraste aos exercícios intensos, que podem aumentar o risco de infecção no trato respiratório superior (30).

Também tem sido reportado que exercícios intensos de duração moderada ( $<60 \mathrm{~min}$ ) e intensidade $\left(<60 \%\right.$ do $\mathrm{VO}_{2 \text { máx. }}$ ) são associados com menores perturbações e menos estresse ao sistema imune do que sessões de exercícios prolongadas e de alta intensidade (30). Outro estudo mostrou que a liberação de cortisol, potencialmente deletéria se encontrada em excesso, parece estar relacionada à intensidade e à duração do exercício. Exercícios contínuos que exigem um alto consumo de oxigênio $\left(\mathrm{VO}_{2}\right.$ máx. $\left.>60 \%\right)$ podem levar a uma leucocitose por um período curto imediatamente após o exercício (32).

Já em um estudo que avaliou os efeitos do exercício resistido nos linfócitos totais, CD4+ e CD8+, de 15 idosas que realizaram exercícios resistidos com cargas de $50 \%$ e $80 \%$ de 1 repetição máxima - RM (carga máxima que pode ser levantada de uma única vez de forma aceitável), observou-se que este tipo de exercício não foi capaz de causar alterações orgânicas nos indivíduos idosos (33). Em uma pesquisa envolvendo 38 mulheres idosas saudáveis submetidas a um treinamento de força durante 12 meses, não se notou nenhuma alteração em seus parâmetros imunológicos (34).

Sabe-se que o processo de envelhecimento é acompanhado por significativas mudanças hormonais. Em particular, ocorre um importante aumento na resistência à insulina à medida que o indivíduo envelhece com significante relevância clínica, por ser considerada como um fator de risco para várias doenças relacionadas ao envelhecimento e estar relacionada a vários componentes individuais da síndrome da fragilidade $(35,36,2)$.

0 exercício físico promove aumento da sensibilidade à insulina e esse benefício pode ser observado tanto com o exercício aeróbio como com o exercício resistido (37). Apesar desse claro benefício, há situações em que o exercício agudo não melhora a sensibilidade à insulina e pode até piorá-la. A sensibilidade à insulina está diminuída após a corrida de maratona, assim como após exercício extenuante e excêntrico, como correr numa ladeira. Uma provável explicação para esse fato é a utilização aumentada e contínua de ácidos graxos como combustível muscular (37).

As reservas de testosterona são mais baixas em idosos (23) e os baixos níveis desse hormônio constituem uma importante causa de sarcopenia, podendo, 
por essa razão, contribuir para o desenvolvimento da fragilidade em homens idosos (38).

Uma resposta acurada de testosterona total e testosterona livre em relação a exercícios resistidos é atenuada em idosos (23). Nem um curto período de treinamento resistido de 10-12 semanas nem um período maior de 21-24 semanas produz um aumento na concentração de testosterona livre e testosterona total (23).

Os efeitos do treinamento resistido em uma variedade de diferentes hormônios têm sido bastante estudados nos últimos anos, porém, a natureza exata dessa relação ainda não está bem compreendida (23).

\section{Orientações para exercício resistido em idosos frágeis}

Em geral, a fragilidade não é contraindicação para exercício, embora modalidades específicas possam ser alteradas para acomodar indivíduos com incapacidade. A idade também não representa fator de impedimento para melhora da função muscular com exercícios resistidos, apresentando melhora comparável àquela observada em adultos jovens (nesses, pode ser maior em função do descondicionamento nos idosos) $(23,24)$.

Um aumento da força muscular após um treinamento de exercício resistido tem sido mais bem documentado em estudos que utilizaram medidas de 1RM ou 3RM quando comparado com medidas isométricas ou isocinéticas. No entanto, apesar de segura, esta prática não é utilizada rotineiramente em razão do potencial de lesão osteomioarticular e da incapacidade dos idosos em realizarem força máxima, ficando a aproximação sucessiva das cargas mais exequível (10). Estudos documentam que idosos podem ter sua força elevada significativamente após um treinamento resistido, com porcentagem variando de menos de $25 \%$ para mais de $100 \%$ (23).

Os componentes de uma prescrição de exercícios resistidos dinâmicos incluem (24):

- Arco de movimento completo (ou máxima amplitude alcançada sem dor) de um exercício;

- Séries de repetições (8-12 repetições por série) sem interrupção;

- A carga utilizada pode ser determinada pela aproximação sucessiva ou, até mesmo (menos utilizada), pela porcentagem de 1RM;
- A velocidade de movimento deve levar de dois a três segundos para levantar o peso (contração concêntrica) e de quatro a seis segundos para baixar o peso (contração excêntrica);

- A duração de uma sessão de treinamento deve ser em torno de 40 minutos.

Um treinamento resistido com frequência de, pelo menos, duas vezes por semana provê um método seguro e efetivo na melhora da força e resistência muscular. Recomenda-se que 8-10 exercícios sejam realizados em dois ou mais dias não consecutivos por semana, sendo utilizando os maiores grupos musculares (12).

Os exercícios devem ser dinâmicos, e não estáticos, englobando os maiores grupos musculares do corpo e utilizando tanto movimentos concêntricos (levantando e empurrando) quanto excêntricos (suaves e controlados no retorno). Os grupos musculares de membros inferiores - como os extensores de joelho e quadril, flexores de joelho, dorsiflexores e flexores plantares - devem ser priorizados, uma vez que são críticos para mobilidade, equilíbrio e prevenção de quedas (24).

Existem algumas recomendações para um treinamento resistido como realizar mínimo de 1 , preferencialmente 2-3 séries por exercícios com 1-2 minutos de descanso entre elas; frequência de 1-3 dias por semana, com mínimo de 48 horas de descanso entre as sessões; respiração normal durante as repetições (não prender a respiração para evitar realizar a manobra de Valsalva - expiração forçada contra a glote fechada) (24).

Há algumas considerações controversas em relação à utilização de procedimentos de triagem antes de iniciar programas de exercícios vigorosos, como testar exercícios. 0 Colégio Americano de Medicina do Esporte recomenda testar exercícios de sintomas limitados antes de realizar exercícios vigorosos ( $>60 \% \mathrm{VO}_{2}$ máx.) em homens com idade $\geq 45$ anos e em mulheres $\geq 55$ anos, em pessoas com dois ou mais fatores de risco cardíaco, em indivíduos com quaisquer sinais/sintomas de doença arterial coronariana, ou aqueles com doenças cardíacas, pulmonares ou metabólicas (12).

Indivíduos sintomáticos ou aqueles com quaisquer doenças cardiovasculares, diabetes, outra doença crônica ou com alguma recomendação médica, devem consultar um médico antes de iniciar um programa de atividades vigoras e/ou intensas (12). 
Embora a habilidade repetida de produzir força muscular em um extenso período possa determinar a variação entre os limites de independência funcional de um idoso, os efeitos do treinamento resistido na resistência muscular são relativamente pouco estudados. 0 aumento da força muscular, secundária a adaptações neurológicas, metabólicas e à hipertrofia, pode explicar o aumento da resistência muscular (23).

\section{Outras pesquisas envolvendo exercícios resistidos em idosos}

Embora poucos estudos sobre os efeitos da prática regular dos ER em população de idosos frágeis tenham sido encontrados na literatura selecionada, diversos estudos apresentam importantes benefícios desta prática em indivíduos senescentes.

Mapeando a importância e eficácia desta modalidade, um elegante estudo realizado por Silva et al. (39) com 61 idosos do gênero masculino, com idades entre 60 e 75 anos, teve como objetivo avaliar o equilíbrio, a coordenação e a agilidade desses indivíduos. Assim, os participantes foram designados aleatoriamente para um grupo de exercícios resistidos com carga progressiva $(n=39)$ ou para um controle submetido a exercícios sem carga $(\mathrm{n}=22)$, e avaliados após o término do treinamento, pela Escala de Equilíbrio de Berg, pelo Teste de Tinetti e pelo Timed Up \& Go. Comparando-se os dois grupos, verificou-se um melhor desempenho estatisticamente significante para o grupo experimental em relação ao controle para os testes Timed Up \& Go. $(\mathrm{p}=0,02)$, Tinetti Total $(p=0,046)$ e Tinetti marcha $(p=0,029)$. Dessa forma, o treinamento resistido mostrou-se favorável na melhora dos desempenhos funcionais e motores de idosos (39).

Em outro estudo, Krinski et al. (40) objetivaram avaliar os efeitos do exercício aeróbio e resistido no perfil antropométrico e respostas cardiovasculares de idosos portadores de hipertensão arterial (HA). A amostra do estudo foi composta de 53 indivíduos de ambos os sexos, todos sedentários, com idade de 64,28 $\pm 4,7$ anos. Assim, foram analisados os níveis de pressão arterial (PA), frequência cardíaca (FC), peso corporal (kg), IMC e percentual de gordura corporal (\%GC), antes, durante e após seis meses de exercícios combinados. 0 protocolo de treinamento foi composto por uma sessão de exercícios, dividida em 20 minutos de atividade aeróbia e 40 minutos de exercícios resistidos dinâmicos, com frequência semanal de três vezes e duração de seis meses. Como principais resultados, foram verificadas diferenças significativas em relação ao \%GC, acompanhadas de uma redução linear na pressão arterial média (PAM) e FC, indicando uma melhora em importantes parâmetros morfofuncionais, o que ratifica a importância da associação dos exercícios aeróbicos e resistidos para a obtenção de resultados positivos.

\section{Considerações finais}

Os idosos constituem uma população bastante heterogênea; apesar de a maior parte deles ser capaz de realizar suas atividades de vida diária, uma pequena parcela é constituída por idosos frágeis que representam os maiores usuários do sistema de saúde, ocupando a maior parte dos leitos destinados a essa faixa etária e ainda utilizando-os por mais tempo. As principais características apresentadas pelos idosos com síndrome da fragilidade são: sarcopenia, distúrbios neuroendócrinos e desregulação do sistema imune.

A atividade física é amplamente discutida na literatura mundial referente a pessoas idosas, e os autores apontam benefícios gerados pela sua prática na qualidade de vida e funcionalidade dessa população. 0 presente estudo se propôs a coletar informações a respeito dos efeitos do exercício resistido na população idosa frágil. 0 treinamento resistido regular é eficaz para reverter ou minimizar os efeitos da sarcopenia. 0 exercício resistido pode levar a um aumento de determinados hormônios sexuais responsáveis pela manutenção do sistema musculoesquelético. Os exercícios de curta duração (como exercícios com carga) aumentam menos a concentração de cortisol no plasma, comparados aos exercícios de longa duração. Os exercícios resistidos não são realizados por um período prolongado sem descanso e representam uma categoria mais segura para aqueles indivíduos que tem o sistema imune prejudicado. 0 exercício físico extenuante pode suprimir a atuação do sistema imune, ao passo que exercícios moderados estimulam o sistema imune e podem ser um tanto responsáveis pela relação dos exercícios e a redução de doenças, podendo oferecer alguma proteção contra malignidades, diminuindo ou evitando alterações fisiológicas apresentadas por idosos com síndrome da fragilidade. 
Considerando-se as evidências apresentadas, e conforme recomendação de entidades reconhecidas nacional e internacionalmente (Sociedade Brasileira de Medicina do Exercício e do Esporte, e American College of Sports Medicine), o treinamento resistido, associado a exercícios aeróbios e de alongamento, deve ser incluído ao menos em duas sessões semanais em uma prescrição de exercícios visando à promoção da saúde e à melhora da aptidão física em indivíduos idosos $(10,23)$.

\section{Referências}

1. Fried LP, Tangen CM, Walston J, Newman AB, Hirsch C, Gottdiener J, et al. Frailty in older adults: evidence for a phenotype. J Gerontol A Biol Sci Med Sci. 2001;56(3): M146-56.

2. Abbatecola AM, Paolisso G. Is there a relationship between insulin resistance and frailty syndrome? Curr Pharm Des. 2008;14(4):405-10. doi.org/10.2174/ 138161208783497750

3. Fried LP, Ferrucci L, Darer J, Williamson JD, Anderson G. Untangling the concepts of disability, frailty, and comorbidity: implications for improved targeting and care. J Gerontol A Biol Sci Med Sci. 2004;59(3):255-63.

4. Chin A, Paw MJ, van Uffelen JG, Riphagen I, van Mechelen W. The functional effects of physical exercise training in frail older people. Sports Med. 2008;38(9):781-93. doi.org/10.2165/00007256-200838090-00006

5. Walston J, Hadley EC, Ferrucci L, Guralnik JM, Newman $A B$, Studenski SA, et al. Research agenda for frailty in older adults: toward a better understanding of physiology and etiology: summary from the American geriatrics society/national institute on aging research conference on frailty in older adults. J Am Geriatr Soc. 2006;54(6):991-1001. doi.org/10.1111/j.15325415.2006.00745.x

6. Walston JD, Schmader KE, Sokol HN. Frailty. UpToDate. Online 71.2; 2009 May [updated 2009 May 12]. [cited 2010 may 13]. http://www.uptodate.com/contents/ frailty.

7. Morley JE, Haren MT, Rolland Y, Kim MJ. Frailty. Med Clin North Am. 2006;90(5):837-47. doi.org/10.1016/j. mcna.2006.05.019
8. Arantes PMM, Alencar MA, Dias RC, Dias JMD, Pereira LSM. Atuação da fisioterapia na síndrome de fragilidade: revisão sistemática. Rev Bras Fisioter. 2009; 13(5):365-75. doi.org/10.1590/S1413-3555200900 5000061

9. Câmara LC, Santarém JM, Wolosker N, Dias RMR. Exercícios resistidos terapêuticos para indivíduos com doença arterial obstrutiva periférica: evidências para a prescrição. J Vasc Bras. 2007;6(3):247-57.

10. Camara LC, Santarém JM, Jacob-Filho W. Atualização de conhecimentos sobre a prática de exercícios resistidos por indivíduos idosos. Acta Fisiatr. 2008;15(4): 257-62.

11. Williams MA, Haskell WL, Ades PA, Amsterdam EA Bittner V, Franklin BA, et al. Resistance exercise in individuals with and without cardiovascular disease: 2007 update: a scientific statement from the American Heart Association Council on Clinical Cardiology and Council on Nutrition, Physical Activity, and Metabolism. Circulation. 2007;116(5):572-84. doi.org/ 10.1161/CIRCULATIONAHA.107.185214

12. Haskell WL, Lee IM, Pate RR, Powell KE, Blair SN, Franklin BA, et al. Physical activity and public health: updated recommendation for adults from the American College of Sports Medicine and the American Heart Association. Circulation. 2007;116(9):1081-93. doi. org/10.1161/CIRCULATIONAHA.107.185649

13. Kushi LH, Byers T, Doyle C, Bandera EV, McCullough M, McTiernan A, et al. American Cancer Society Guidelines on Nutrition and Physical Activity for cancer prevention: reducing the risk of cancer with healthy food choices and physical activity. CA Cancer J Clin. 2006;56(5): 254-81. doi.org/10.3322/canjclin.56.5.254

14. da Nóbrega ACL, de Freitas EV, de Oliveira MAB, Leitão MB, Lazzoli JK, Nahas RM, et al. Posicionamento Oficial da Sociedade Brasileira de Medicina do Esporte e da Sociedade Brasileira de Geriatria e Gerontologia: Atividade Física e Saúde no Idoso. Rev Bras Med Esporte 1999;5(6):207-11.

15. Borst SE. Interventions for sarcopenia and muscle weakness in older people. Age and ageing. 2004;33(6): 548-55. doi.org/10.1093/ageing/afh201

16. Narici MV, Maffulli N. Sarcopenia: characteristics, mechanisms and functional significance. Br Med Bull. 2010;95:139-59. doi.org/10.1093/bmb/ldq008 
17. Patel HP, Syddall HE, Martin HJ, Stewart CE, Cooper C, Sayer AA. Hertfordshire sarcopenia study: design and methods. BMC Geriatr. 2010;10:43. doi.org/10. 1186/1471-2318-10-43

18. Rexach JAS. Consecuencias clínicas de la sarcopenia. Nutr Hosp. 2006;21(Supl. 3):46-50.

19. Volpi E, Nazemi R, Fujita S. Muscle tissue changes with aging. Curr Opin Clin Nutr Metab Care. 2004;7(4): 40510. doi.org/10.1097/01.mco.0000134362. 76653.b2

20. Serra Rexach JA, Ruiz JR, Bustamante-Ara N, Villarán MH, Gil PG, Sanz Ibáñez MJ, et al. Health enhancing strength training in nonagenarians (STRONG): rationale, design and methods. BMC Public Health. 2009;9:152. doi. org/10.1186/1471-2458-9-152

21. Galvão DA, Ewyon RU, Taaffe DR. Anabolic responses to resistance training in older men and women: a brief review. J Aging Phys Act. 2005;13(3):343-58.

22. Melov S, Tarnopolsky MA, Beckman K, Felkey K, Hubbard A. Resistance exercise reverses aging in human skeletal muscle. PLoS One. 2007;2(5):e465. doi.org/10.1371/journal.pone.0000465

23. American College of Sports Medicine, Chodzko-Zajko WJ, Proctor DN, Fiatarone Singh MA, Minson CT, Nigg CR, et al. American College of Sports Medicine position stand. Exercise and physical activity for older adults. Med Sci Sports Exerc. 2009;41(7):1510-30.

24. Taafe DR. Sarcopenia - exercise as a treatment strategy. Aust Fam Physician. 2006;35(3):130-4.

25. Binder EF, Yarasheski KE, Steger-May K, Sinacore DR, Brown M, Schechtman KB, et al. Effects of progressive resistance training on body composition in frail older adults: results of a randomized, controlled trial. J Gerontol A Biol Sci Med Sci. 2005;60(11):1425-31.

26. Hurme M, Paavilainen PM, Pertovaara M, Jylhä M, Karhunen PJ, Hervonen A, et al. IgA levels are predictors of mortality in Finnish nonagenarians. Mech Ageing Dev. 2005;126(6-7):829-31. doi.org/10.1016/j. mad.2005.02.005

27. Chin, A Paw MJ, de Jong N, Pallast EG, Kloek GC, Schouten EG, Kok FJ. Immunity in frail elderly: a randomized controlled trial of exercise and enriched foods. Med Sci Sports Exerc. 2000;32(12):2005-11.
28. Friedrich MJ. Exercise may boost aging immune system. JAMA. 2008;299(2):160-1.

29. Silveira EM, Rodrigues MF, Krause MS, Vianna DR, Almeida BS, Rossato JS, et al. Acute exercise stimulates macrophage function: possible role of NF-kappaB pathways. Cell Biochem Funct. 2007;25(1):63-73. doi.org/10.1002/cbf.1365

30. Malaguarnera L, Cristaldi E, Vinci M, Malaguarnera $M$. The role of exercise on the innate immunity of the elderly. Eur Rev Aging Phys Act. 2008;5(1):43-9. doi. org/10.1007/s11556-007-0028-8

31. Keylock KT, Lowder T, Leifheit KA, Cook M, Mariani RA, Ross K, et al. Higher antibody, but not cell-mediated, responses to vaccination in high physically fit elderly. J Appl Physiol. 2007;102(3):1090-8. doi.org/10. 1152/japplphysiol.00790.2006

32. Leandro C, Nascimento E, Manhães-de-Castro R, Duarte J, de-Castro C. Exercício físico e sistema imunológico: mecanismos e integrações. Rev Port Ciênc Desporto. 2002;2(5):80-90.

33. Ávila WRM. Efeitos agudos do exercício resistido sobre linfócitos totais, CD4 e CD8 de Idosas. (dissertação). Universidade Católica de Brasília - UCB/DF; Brasília; 2006.

34. Raso V. Efeitos de doze meses de um programa de exercícios com pesos em parâmetros imunológicos de mulheres idosas clinicamente saudáveis. (dissertação) Faculdade de Medicina da Universidade de São Paulo - USP, São Paulo; 2005.

35. Geroldi C, Frisoni GB, Paolisso G, Bandinelli S, Lamponi $\mathrm{M}$, Abbatecola AM, et al. Insulin resistance in cognitive impairment: the In CHIANTI study. Arch Neurol. 2005;62(7):1067-72. doi.org/10.1001/archneur. 62.7.1067

36. Abbatecola AM, Ferrucci L, Ceda G, Russo CR, Lauretani F, Bandinelli S, et al. Insulin resistance and muscle strength in older persons. J Gerontol A Biol Sci Med Sci. 2005;60(10):1278-82.

37. Cialoc EG, Guimarães GV. Exercício físico e síndrome metabólica. Rev Bras Med Esporte. 2004;10(4): 319-24. 
38. Srinivas-Shankar U, Roberts SA, Connolly MJ, O'Connell MD, Adams JE, Oldham JA, et al. Effects of testosterone on muscle strength, physical function, body composition, and quality of life in intermediate-frail and frail elderly men: a randomized, double-blind, placebocontrolled study. J Clin Endocrinol Metab. 2010;95(2): 639-50.

39. Silva A, Almeida JM, Cassilhas RC, Cohen M, Peccin MS, Tufik S, de Mello MT, et al. Equilíbrio, coordenação e agilidade de idosos submetidos à prática de exercícios físicos resistidos. Rev Bras Med Esporte. 2008;14(2):88-93. doi.org/10.1590/S151786922008000200001
40. Krinski K, Elsangedy, HM, Nardo N Jr, Soares IA. Efeito do exercício aeróbio e resistido no perfil antropométrico e respostas cardiovasculares de idosos portadores de hipertensão. Acta Sci Health Sci. 2006;28(1): 71-5.

Recebido: 20/02/2010

Received: 02/20/2010

Aprovado: 01/08/2011

Approved: 08/01/2011 